\title{
EMD-Based Short-term load forecasting
}

\author{
Guo Shu-Qin ${ }^{1}$, Ruan Lin $^{2}$, \\ Institute of Electrical Engineering, Chinese Academy of \\ Sciences, Information Equipment Application of \\ Evaporating Cooling Technology Incubation and \\ Research Center \\ Beijing, China \\ sqguo@mail.iee.ac.cn \\ rosaline@mail.iee.ac.cn
}

\begin{abstract}
Based on the characteristics of power load series such as periodic randomness and instability, A new method for short-term load forecasting was presented in this paper. Firstly, the power load series was decomposed into intrinsic mode functions (IMF). Then, according to the traits of each IMF, System Identification Toolbox of Mat lab 7.0 was used in selecting models (AR, MA or ARMA), setting orders, realizing forecasting and reconstructing the predictive values. The simulation was carried out with the data from a grid and the results showed that this method could achieve a high level of precision in forecasting.
\end{abstract}

Keywords- EMD, Time series, short-term load forecasting, System Identification Toolbox

\section{INTRODUCTION}

Power system load is a very complex nonlinear system. It is changing with time. The short- term load with cyclical changes generally embraces the characteristics of randomness. To improve the quality of short-term load forecasting is helpful to arrange the starting and breaking of generation units, formulate reasonable maintenance scheduling, maintain the safe operation of power network, reduce power generation cost, and enhance the level of transactions for electric energy market, thus developing the system's security while ensuring economic and social benefits. That is why the short-term load forecasting study is of great significance.

A lot of new methods for short-term load forecasting have been put forward over the past decades, such as artificial neural network, support vector machine prediction, expert system, the combination of model prediction and so forth. These methods have some advantages in reducing randomness and uncertainty while showing some shortages [1]. This paper used the method of empirical mode decomposition combining with the traditional model of timeseries to forecast short-term load to a grid data.

\section{BASIC PRINCIPLES}

\section{A. Empirical Mode Decomposition}

Empirical mode decomposition (EMD) is an adaptive nonlinear decomposition technique for analyzing nonlinear

\author{
Dong Hai-Hong ${ }^{3}$, Li Zhen-Guo ${ }^{3}$,Liu Fei-Hui ${ }^{3}$, and \\ Cao Rui ${ }^{3}$ \\ Institute of Electrical Engineering, Chinese Academy of \\ Sciences, Information Equipment Application of \\ Evaporating Cooling Technology Incubation and \\ Research Center \\ Beijing, China \\ d_haihong@mail.iee.ac.cn
}

and non-stationary signals. It has higher SNR(signal to noise ratio). In essence it is the signal for a smooth process. The result is that the fluctuations or trends in different scales have been decomposed, and a series of data sequence can be acquired using scales with different characteristics. Each sequence can be defined as an Intrinsic Mode Function(IMF), and the frequency components of each contained IMF not only relate with frequencies but can change with the signal itself. Intrinsic mode function is to satisfy the following conditions for a class of functions:

(1) The number of extrema and zero-crossings of the function must be equal or differ by no more than one.

(2) The mean at any Point of the envelope defined by the local maxima and local minima is zero.

Empirical mode decomposition algorithm can be described as follows [2]:

(1) Identify all the local extreme from the given signal $\mathrm{x}(\mathrm{t})$, and then connect all the local maxima by cubic spine line as the upper envelope. Repeat the previous step for the local minima to produce the lower envelope. The upper and lower envelope should cover the signal between them. Their mean is designated as $\mathrm{m}_{1}(\mathrm{t})$, and the difference between the signal $\mathrm{x}(\mathrm{t})$ and $\mathrm{m}_{1}(\mathrm{t})$ is the first component $\mathrm{h}_{1}(\mathrm{t})$, i.e. ,.

$$
\mathrm{h}_{1}(\mathrm{t})=\mathrm{x}(\mathrm{t})-\mathrm{m}_{1}(\mathrm{t}) \text {. }
$$

(2) To eliminate riding waves and make the wave profiles more symmetric, the sifting process of Eq.(1) should be repeated as many times as required to reduce the extracted signal to an IMF. In the next step, $h_{1}(t)$ is treated as the signal, and

$$
\mathrm{h}_{11}(\mathrm{t})=\mathrm{h}_{1}(\mathrm{t})-\mathrm{m}_{11}(\mathrm{t}) \text {. }
$$

After repeated siftings in this manner up to $\mathrm{k}$ times, $\mathrm{h}_{1} \mathrm{k}(\mathrm{t})$ becomes an IMF; that is,

Then, it is designated as

$$
\mathrm{h}_{1 \mathrm{k}}(\mathrm{t})=\mathrm{h}_{1(\mathrm{k}-1)}(\mathrm{t})-\mathrm{m}_{1 \mathrm{k}}(\mathrm{t})
$$

$$
\mathrm{c}_{1}(\mathrm{t})=\mathrm{h}_{1 \mathrm{k}}(\mathrm{t}) \text {. }
$$

the first IMF component from the signal.

(3) Generally speaking, $c_{1}(t)$ contains the finest scale or the shortest period component of the signal. Separating $c_{1}(t)$ from the rest of the signal by

$$
r_{1}(t)=x(t)-c_{1}(t) \text {. }
$$


Then the residue of the signal $r_{1}(t)$, which contains longer period variations in the signal, is obtained. Treating $\mathrm{r} 1(\mathrm{t})$ as a new signal and subjecting it to the same sifting process as described above, the second IMF c2(t) is obtained. This procedure can be repeated with all the subsequent $r j(t)$, and a series of IMFs $\operatorname{ci}(\mathrm{t})(\mathrm{i}=1,2, \ldots \ldots, \mathrm{n})$, and the final residue $\mathrm{rn}(\mathrm{t})$ is consequently extracted. Summing up all the IMFs and the final residue $\mathrm{rn}(\mathrm{t})$, the given signal $\mathrm{x}(\mathrm{t})$ is reconstructed by

$$
x(t)=\sum_{i=1}^{n} c_{i}(t)+r_{n}(t) .
$$

The variable amplitude and the instantaneous frequency have not only greatly improved the efficiency of the expansion, , but also enable the expansion to accommodate nonlinear and no stationary signals.

\section{B. Linear time series model}

Time series changes randomly in sequence. Time series model reveals the statistical relationship from various time series of internal relations and between the various time series. The relatively sophisticated linear time series models mainly include: Auto Regressive Model(AR)、 Moving Average Model(MA) and Auto Regressive Integrated Moving Average Model(ARMA)[3].

We care the linear relationship of the random variable $x(t)$ at different moments for linear time series $\mathrm{x}(\mathrm{t})$. Autocorrelation function (ACF) and partial autocorrelation function (PACF) have provided important information for the time series of related structures, and plays an active role in model identification and estimation. Autocorrelation function describes the degree of linear dependency between $\mathrm{x}_{(\mathrm{i}+\mathrm{k})}$ and $\mathrm{x}_{\mathrm{i}}$,; Partial autocorrelation functions are described in the relevance of residual after $\mathrm{x}_{(\mathrm{i}+\mathrm{k})}$ and $\mathrm{x}_{\mathrm{i}}$, respectively, for linear regression of $\mathrm{x}_{(\mathrm{i}+1)}, \cdots, \mathrm{x}_{(\mathrm{i}+\mathrm{k}-1)}$. For example, the autocorrelation functions of $\mathrm{P}$-order autoregressive process $(\mathrm{AR}(\mathrm{P}))$ are tailing, but tailing but their partial autocorrelation functions are bobtailed after lagging $\mathrm{P}$ steps. The autocorrelation functions of moving average process (MA(q))is bobtailed after lagging q steps, but their partial autocorrelation functions are tailing. The autocorrelation and partial autocorrelation functions are tailing.

As mentioned above, power load is a non-stationary time series. The premise of using linear model to do model identification and prediction is to request data sequences $\mathrm{x}(\mathrm{t})$ are stable. In this paper, empirical mode decomposition method has been used to divide signal into 8 smooth components.

\section{BUILDING PREDICTION MODEL}

\section{A. Data Preprocessing}

Electricity load demand recorded every half hour, from 1997 to 1998.

The task of this paper is to predict the maximum daily value of electrical loads in January 1999 using those data.
Evaluation criteria include mean absolute percentage error(MAPE):

$$
\text { MAPE }=\frac{1}{\mathrm{n}} \sum_{\mathrm{i}=1}^{\mathrm{n}}\left|\frac{\mathrm{L}_{\mathrm{i}}-\hat{\mathrm{L}}_{\mathrm{i}}}{\mathrm{L}_{\mathrm{i}}}\right| * 100 \% . \quad \mathrm{i}=1,2, \ldots, 31
$$

and maximum error(ME):

$$
\mathrm{ME}=\max \left|\mathrm{L}_{\mathrm{i}}-\widehat{\mathrm{L}}_{\mathrm{i}}\right| . \quad \mathrm{i}=1,2, \ldots, 31
$$

This article defines the daily load error(ERR):

$$
\operatorname{ERR}=\mathrm{L}_{\mathrm{i}}-\widehat{\mathrm{L}}_{\mathrm{i}} .
$$

Where $\mathrm{L}_{\mathrm{i}}$ and $\widehat{\mathrm{L}}_{\mathrm{i}} \stackrel{\Lambda}{L}_{i}$ are the real value and the predicted value of maximum daily electrical load on the $i$ day of the year 1999 respectively, and $\mathrm{n}$ is the number of days in January 1999.

First of all, the maximum daily load is calculated using the historical data of two years, and 730 data are got. Then, we can consider the loads as a time series. And, we linearly scale all load data to [0 1]. Then the same calculation is done to the data in January 1999, and 31data are got, which is used as the comparison value of the final prediction results.

\section{B. Empirical Mode Decomposition}

The 730 data have been decomposed by EMD. The electric load data are decomposed into seven modes and one trend, showed in Figure 1.

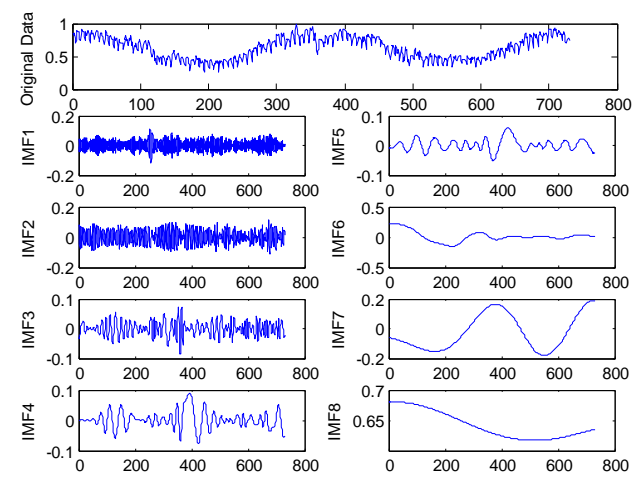

Figure 1. The maximum daily load data from 1997 to 1998

decomposed into seven modes and one trend by the EMD The eight components give prominence the local features of electricity load signal from figure 1. Here IMF1、IMF2 and IMF3 are the high frequency components. They show randomness of original load sequence; IMF4 and IMF5 have periodicity and they are the periodic terms; IMF6、IMF7and IMF8 are the trend terms. The amplitude of IMF8 is in agreement with the amplitude of original load sequence. Therefore, the component of IMF8 plays a decisive role in load variation, and the other components only effect the fluctuation of load curve. 


\section{C. model parameters identification}

First of all, we must calculate the auto-correlation coefficient and the partial autocorrelation coefficient of the each IMF components at $95 \%$ confidence interval. So the model can be preliminarily ascertained.

We calculate the auto-correlation coefficient by function autocorr, and the partial autocorrelation coefficient by function parcorr. The results are shown in Figure 2(taking IMF3、IMF5、IMF8 as an example).

The auto-correlation coefficient of IMF3 is tailing, and the partial autocorrelation coefficient of IMF3 is bobtailed from Figure 2-a, preliminary being judged to the MA(q) model. The auto-correlation coefficient and the partial autocorrelation coefficient of IMF5 are all bobtailed from Figure 2-b, preliminary being judged to the $\operatorname{ARMA}(p, q)$ model. The auto-correlation coefficient of IMF8 is bobtailed, and the partial autocorrelation coefficient of IMF8 is tailing from Figure 2-c. preliminary being judged to the $\operatorname{AR}(p)$ model. The other components are judged by the same method.

This paper adopts AIC function of MATLAB7.0 to fit each component. The model which AIC value reaches the minimum is the best model, and the values of $p$ and $q$ are the model order. The results are shown in Table1.
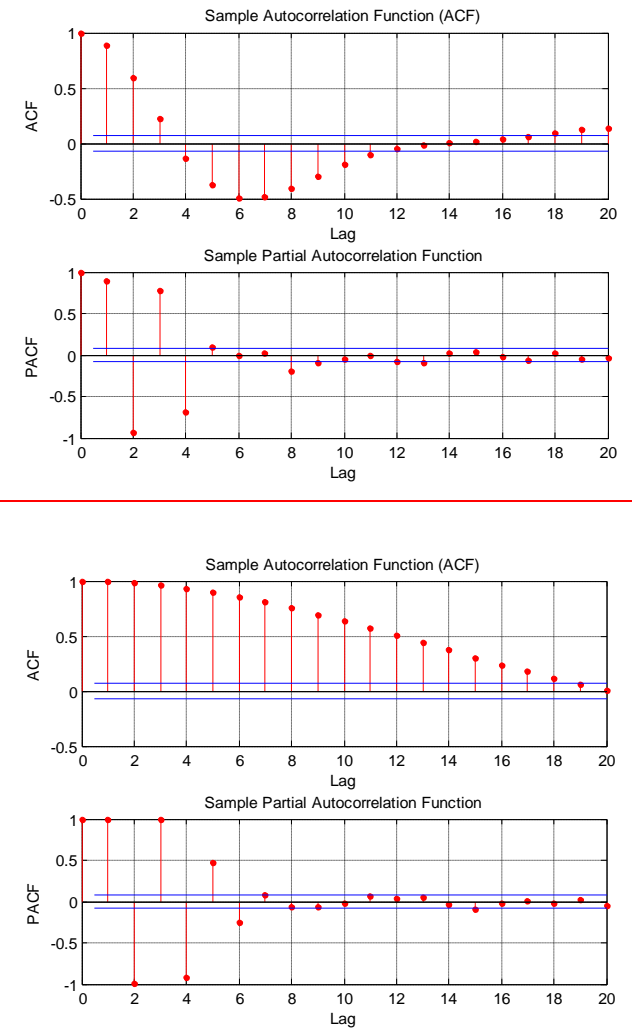
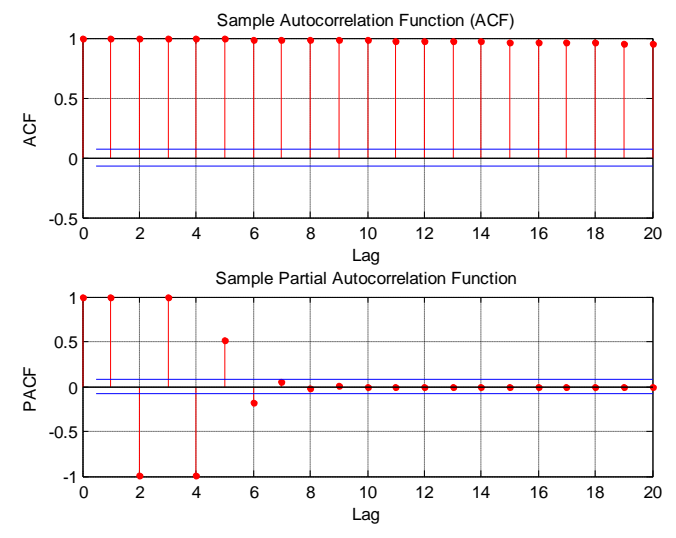

Figure 2. The ACF and PACF

IMF3: AIC $=-12.6107$,

Discrete-time IDPOLY model: $\mathrm{y}(\mathrm{t})=\mathrm{C}(\mathrm{q}) \mathrm{e}(\mathrm{t})$

$C(q)=1+3.162 q^{-1}+5.63 q^{-2}+7.228 q^{-3}+6.946 q^{-4}+4.668 q^{-5}+1.199$ $\mathrm{q}^{-6}-2.231 \mathrm{q}^{-7}-4.617 \mathrm{q}^{-8}-5.554 \mathrm{q}^{-9}-5.148 \mathrm{q}^{-10}-3.896 \mathrm{q}^{-11}-2.415 \mathrm{q}^{-12}-1.191 \mathrm{q}^{-13}$ $0.4208 q^{-14}-0.07893 q^{-15}$

IMF5: AIC $=-23.0337$

Discrete-time IDPOLY model: $A(q) y(t)=C(q) e(t)$

$A(q)=1-2.882 q^{-1}+2.784 q^{-2}-0.9007 q^{-3}$

$\mathrm{C}(\mathrm{q})=1+1.586 \mathrm{q}^{-1}+1.543 \mathrm{q}^{-2}+1.325 \mathrm{q}^{-3}+1.115 \mathrm{q}^{-4}+0.8645 \mathrm{q}^{-5}+$ $0.5369 q^{-6}+0.3079 q^{-7}+0.1866 q^{-8}+0.1126 q^{-9}+0.05225 q^{-10}$

IMF8: AIC $=-44.4869$

Discrete-time IDPOLY model: $\mathrm{A}(\mathrm{q}) \mathrm{y}(\mathrm{t})=\mathrm{e}(\mathrm{t})$

$A(q)=1-4.613 q^{-1}+8.632 q^{-2}-8.406 q^{-3}+4.553 q^{-4}-1.352 q^{-5}+0.186 q^{-6}$

TABLE I. RESULTS OF ORDER DETERMINATION FROM IMF1 TO IMF8

\begin{tabular}{|c|l|c|c|}
\hline IMF1 & IMF2 & IMF3 & IMF4 \\
\hline$A R M A(6,13)$ & $A R M A(12,11)$ & $M A(15)$ & $A R M A(1,20)$ \\
\hline IMF5 & IMF6 & IMF7 & IMF8 \\
\hline$A R M A(3,10)$ & $A R M A(3,15)$ & $A R M A(3,22)$ & $A R(6)$ \\
\hline
\end{tabular}

\section{Diagnostic Test}

Using residual analysis to test that whether the residual of the model is white noise or not. This paper adopts reside function to calculate predict error of the model. The autocorrelation coefficient and the partial autocorrelation coefficient are shown in Figure 3.

According to the analysis, the residuals of the components from IMF1 to IMF8 are white noise. All the models meet demands, so we can continue to implement the next forecasting. 

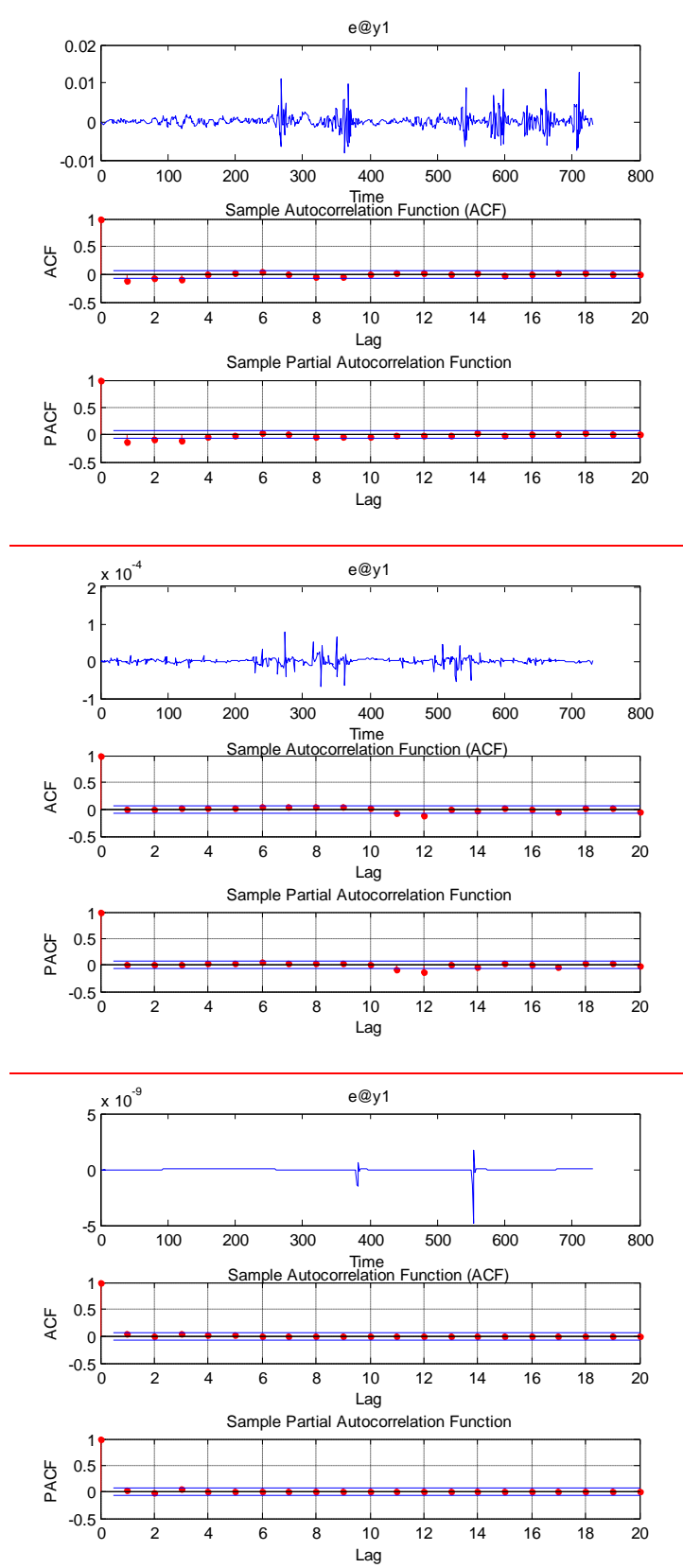

Figure 3. The residuals and ACF, PACF figure

\section{PRediction Results AND ANALYSIS}

This paper adopts predict function to predict new data with the eight models having been established. Restriction the eight group results to get the final results. Prediction curve and prediction error cure are shown in Figure4.
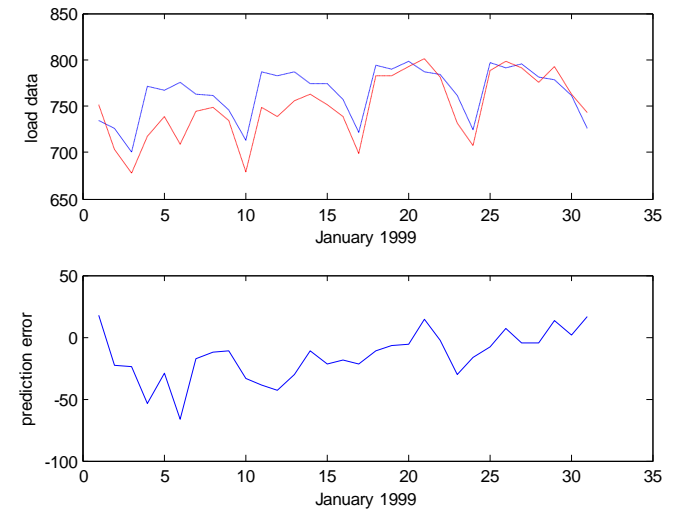

Figure 4. Prediction result and error

The dotted line is predictive value, and the solid line is real value. The values of MAPE and ME are shown in Table 2. The prediction errors of the first two weeks are larger than those of the two weeks later. The prediction errors of the high-frequency components are larger than those of the lowfrequency components. And the frequency is lower, the prediction error is smaller. The prediction error of IMF8 approach zero.

TABLE II. A COMPARISON OF PREDICTION ERROR

\begin{tabular}{|l|l|l|}
\hline Error & Suggested method & Direct prediction \\
\hline MAPE (\%) & 2.73 & 8.66 \\
\hline ME & 66.1564 & 167.6845 \\
\hline
\end{tabular}

If the ARMA model is directly used to predict the new data, the prediction results are shown in Figure 5. It is obviously that the prediction result is not good than the result using Empirical mode decomposition method.
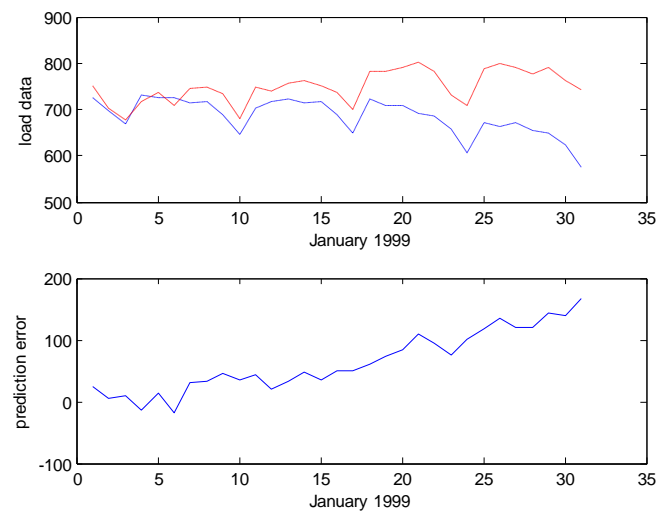

Figure 5. Prediction result and error

\section{CONCLUSIONS}

This paper adopts empirical mode decomposition method in combination with linear time series model to implement short-term load forecasting. The power load itself is a nonlinear, non-stationary process. Empirical mode 
decomposition method can only decompose the nonstationary signals into $\mathrm{n}$ stationary signals. In other words, both linear and nonlinear factors exist in the IMF components by empirical mode decomposition. This paper completely adopts linear time series to modeling and prediction. There are low computation amount and fast speed in this method. The prediction accuracy is high to the lowfrequency component. For example, the component from IMF5 to IMF8 in this paper; but which is relatively low to the high-frequency component (from IMF1 to IMF4). At present, some scholars use EMD in combination with SVM (Support Vector Machine) [4] or in ANN (Artificial Neural Network)[5] to implement the short-term load forecasting, and active good effect. So it is worth studying that using ANN or SVM to predict high-frequency component by empirical mode decomposition.

\section{REFERENCES}

[1] Tang Jieming, Liu Junyong, Liu Youbo. "New method of power system short-term load forecasting" [J].Sichuan electric power technology, Vol 31,No 1,pp 61-65,2008.

[2] Yu Weikai, "The eroretical study and application of time-frequency analysis method based on EMD'[J].YanShan University,2006.

[3] Fan Jianqing, Yao Qiwei(writer), Chen Min(translator). "The modeling and application of nonlinear time series"[M]. Bei Jing: the higher education press, 2005.

[4] Duan Qichang, Rao Zhibo,Huang Dawei,Lin Sen. "Short-term load forecasting based on EMD and PSO-SVM"[J]. Control Engineering of China, No 5. 2012.

[5] Peng Shenggang, Li Jide. "Time series used in short time forcasting" [J]. Journal of Shandong electric power college, No 4,pp 51-54, 2012. 